\title{
Interactive comment on "Experimental
} determination of Henry's law constants of difluoromethane (HFC-32) and the salting-out effects in aqueous salt solutions relevant to seawater" by Shuzo Kutsuna

\section{S. Kutsuna}

s-kutsuna@aist.go.jp

Received and published: 8 February 2017

I am sorry because Eqs. (21) and (22) and the corresponding equation in the abstract were mistyped as indicated below.

Eq. (21) should be represented as follows: 
$\ln \left(K_{e q}^{S}(T)\right)=-49.7122+77.7018 \times(100 / T)+19.1379 \times \ln (T / 100)+[-0.2261+$ $0.5176 \times(100 / T)] \times S^{0.5}+[0.0362-0.1046 \times(100 / T)] \times S$

In Abstract section, 1st block, lines 6-7, the equation corresponding to Eq. (22) should be represented as follows:

$\ln \left(K_{e q}^{S}(T)\right)=-49.7122+77.7018 \times(100 / T)+19.1379 \times \ln (T / 100)+[-0.2261+$ $0.5176 \times(100 / T)] \times S^{0.5}+[0.0362-0.1046 \times(100 / T)] \times S$

Interactive

comment

The above corrections are needed because of the mistype of a sign of the right hand of Eq. (21) and the mistype of ( $T / 100)$ instead of the correct term of $(100 / T)$ for Eq. (21).

Data analysis had been performed by the correct Equations thorough the manuscript; hence, the parameters determined and the results estimated in the manuscript were correct.

I am sorry for my mistake in Eq. (21), Eq. (22) and the Equation corresponding to Eq. (22) in Abstract section.

Interactive comment on Atmos. Chem. Phys. Discuss., doi:10.5194/acp-2017-58, 2017. 